\title{
Correlações entre Habilidades Sociais Educativas dos Professores, Burnout e Relação Professor-Aluno
}

\section{Correlations of Teachers' Social Educative Skills, Burnout and the Teacher-Student Relationship}

\section{Correlaciones de las Habilidades Sociales Educativas de los Profesores, del Burnout y la Relación Profesor-Alumno}

\author{
Ana Maria Nunes El Achkar* \\ Universidade Salgado de Oliveira - UNIVERSO, Niterói, Rio de Janeiro, Brasil
}

Vanessa Barbosa Romera Leme**

Universidade do Estado do Rio de Janeiro - UERJ, Rio de Janeiro, Brasil

\section{Adriana Benevides Soares***}

Universidade Salgado de Oliveira - UNIVERSO, Niterói, Rio de Janeiro, Brasil

\author{
Maria Angela Mattar Yunes**** \\ Centro Universitário La Salle Canoas - Unilasalle, Canoas, Rio Grande do Sul, Brasil
}

\begin{abstract}
RESUMO
Este estudo teve por objetivo correlacionar as habilidades sociais educativas dos professores (HSE), o Burnout e a relação professor-aluno. Participaram 400 professores $(M=36,08$ anos, $D P=9,94)$, de ambos os sexos (348 mulheres e 52 homens), os quais lecionavam no 70, $8^{\circ}$ e 90 ano do Ensino Fundamental, provenientes de oito escolas, sendo quatro públicas e quatro particulares, situadas em cidades do Estado do Rio de Janeiro. Os professores responderam ao Questionário com informações demográficas e nível socioeconômico, à Escala da Relação Professor-Aluno (IHSE-DelPrette), ao Inventário de Habilidades Sociais Educativas (IHSE-Del-Prette) e ao Inventário de Burnout de Maslach (MBI). Os resultados indicaram que as HSE dos professores se associaram positivamente com a relação professoraluno e negativamente com o Burnout, sendo esse correlacionado negativamente com a relação professor-aluno. O modelo de regressão evidenciou que as HSE dos professores têm maior poder preditivo sobre as relações entre professores e alunos nos Anos Finais do E.F. Conclui-se que intervenções com foco na aprendizagem de HSE dos professores podem contribuir para a prevenção de Burnout, assim como promover o desenvolvimento acadêmico e socioafetivo de seus alunos.
\end{abstract}

Palavras-chave: intervenção, autoeficácia, burnout, HSE.

\section{ABSTRACT}

This study aimed to correlate teacher's social educative skills, Burnout and teacher-student relationship. 400 teachers $(M=36.08$ years old, $S D=9.94)$ 
of both genders (348 females and 52 males) that taught grade 7, 8 and 9 students of four private and four public middle schools, situated in cities of the state of Rio de Janeiro, participated of the study. The teachers answered a demographic and socio-economic questionnaire, a Teacher-Student Scale (TSE), a Social Skill Educative Inventory (SSEI) and a Maslach Burnout Inventory (MBI). Results indicated that teachers' social educative skills correlated positively with the teacher-student relationship but negatively with Burnout, which correlated negatively with the teacher-student relationship. Furthermore, the regression model evidenced that the teachers' social educative skills have stronger predictive power over the relationships between teachers and students in final middle-school grades. Thus, it is possible to conclude that interventions with a focus on teacher's social educative skills learning may contribute to Burnout prevention, as well as promote academic and socio-affective development of their students.

Keywords: interventions, self-efficacy, burnout, ESS.

\section{RESUMEN}

Este estudio tuvo por objetivo correlacionar las habilidades sociales educativas de los profesores (HSE), del Burnout y la relación profesoralumno. Participaron 400 profesores $(M=36,08$ años, $D P=9,94)$, de ambos sexos ( 348 mujeres y 52 hombres) que enseñaban el $70,8^{\circ}$ y $9^{\circ}$ año de la Enseñanza Fundamental, provenientes de ocho escuelas, siendo cuatro públicas y cuatro particulares, que se encuentra en ciudades del Estado de Río de Janeiro. Los profesores respondieron al Cuestionario con informaciones demográficas y nivel socioeconómico, a la Escala de la Relación Profesor-Alumno (ERPA), al Inventario de Habilidades Sociales Educativas (IHSE) y al Inventario de Burnout de Maslach (MBI). Los resultados indicaron que las HSE de los profesores asociaron positivamente con la relación profesor-alumno y negativamente con el burnout, siendo ese correlacionado negativamente con la relación profesor-alumno. La plantilla de regresión evidencio que las HSE de los profesores tienen mayor poder predictivo sobre las relaciones entre profesores y alumnos los años finales del E.F. Se concluye que intervenciones con foco en el aprendizaje de HSE de los profesores pueden contribuir para la prevención de Burnout, así como promover el desarrollo académico y socio afectivo de sus alumnos.

Palabras clave: intervenciones, auto eficácia, burnout, HSE.

\section{Introdução}

Nas últimas duas décadas, um grande número de investigações tem sido realizado a respeito da importância das relações afetivas entre professores e alunos para o desenvolvimento socioafetivo e cognitivo dos estudantes (Cooper, 2014; Dotterer \& Lower, 2011; Machado, Yunes, \& Silva, 2014; Mattos, Pérez, Almada, \& Castro, 2013; Meireles, 2009; Paiva \& Del Prette, 2009). Estudos têm estabelecido que as relações positivas entre professor-aluno são responsáveis por vários indicadores de sucesso escolar, não só em termos acadêmicos, mas também no que se refere ao funcionamento socioafetivo dos mesmos (Cooper, 2014; Dotterer \& Lower, 2011; Mattos et al., 2013; Meireles, 2009; Paiva \& Del Prette, 2009). 
De modo geral, as pesquisas indicam que, dependendo do tipo das relações interpessoais e do apoio social recebido nesses ambientes, processos de resiliência podem ser desenvolvidos e vulnerabilidades superadas. Nesse sentido, outros estudos destacam ser essencial a figura do professor como um facilitador e/ou mediador no cotidiano escolar para a efetividade dos processos de ensino-aprendizagem (Achkar, 2013; Fajardo, 2015; Facci, Tessaro, Leal, Silva, \& Roma, 2007; Machado et al., 2014; Soares, Naiff, Fonseca, Cardozo, \& Baldez, 2009).

Conforme Soares et al. (2009), o docente é responsável não apenas por inserir e manter o estudante no contexto acadêmico, mas, especialmente, deve estimular sua curiosidade e seu interesse pelo conhecimento, propiciando desenvolvimento intelectual e interpessoal. Portanto, o ofício do educador precisa intencionar algo mais do que a mera transmissão e comprovação de conteúdos acadêmicos. A sala de aula não se configura apenas como local de transmissão de saberes, mas, sobretudo, em um espaço de obtenção e fortalecimento de valores éticos-morais que poderão facilitar o desenvolvimento de relações interpessoais (Del Prette \& Del Prette, 2008; Facci et al., 2007; Machado et al., 2014; Soares et al., 2009).

Em relação à prática docente, o contexto do Ensino Fundamental (E.F.) tem sido caracterizado por riscos tais como: excesso de carga horária, salários inadequados, turmas numerosas e de violência intra e extramuros da escola (Dalagasperina \& Monteiro, 2014; DoménechBetoret, Lloret-Segura, \& Gómez-Artiga, 2015; Mesquita, Gomes, Lobato, Gondim, \& Souza, 2013). Apesar disso, a atuação docente pode envolver habilidades interpessoais que atenuam a sobrecarga de trabalho em professores propensos a sensações conflitantes e estressantes, ou manifestações de Burnout. Consequentemente, os resultados evidenciam melhoras na qualidade da relação entre o professor e seus alunos (Carlotto, Dias, Batista, \& Diehl, 2015; Dalagasperina \& Monteiro, 2014; Doménech-Betoret et al., 2015; Mesquita et al., 2013). O Burnout é um termo inglês que significa desgaste ou exaustão. Tornou-se conhecido a partir da década de 1970, sendo primeiramente documentado entre as profissões de militares, médicos e bombeiros (Dalagasperina \& Monteiro, 2014; Maslach, Schaufeli, \& Leiter, 2001).

Pesquisas salientam a necessidade de que o trabalho docente seja saudável, confiante e eficaz e, que a partir dele, os educadores se disponham e se sintam motivados a construir processos de resiliência com os estudantes (Achkar, 2013; Fajardo, 2015; Henderson \& Milstein, 2003; Noltemeyer, 2013; Yunes, Pietro, Silveira, Juliano, \& Garcia, 2015). Após mais de três décadas de estudos, resiliência deixou de ser definida como traço ou qualidade de personalidade individual e tem sido compreendida como processos e produto de interações dinâmicas entre situações de risco e de proteção que 
resultam em transformações positivas em indivíduos, grupos e comunidades (Coimbra \& Fontaine, 2015; Fletcher \& Sarkar, 2013; Juliano \& Yunes, 2014; Masten, 2014; Noltemeyer \& Bush, 2013). Processos de resiliência podem ter origem em relações familiares, escolares e comunitárias benéficas que oportunizam um satisfatório apoio social (Fletcher \& Sarkar, 2013; Garcia, Brino, \& Williams, 2009; Juliano \& Yunes, 2014; Fernandes \& Leme, 2014; Noltemeyer, 2013; Noltemeyer \& Bush, 2013; Ungar, 2012). Desse modo, com o objetivo de investigar variáveis que podem promover ou dificultar processos de resiliência em contexto escolar e, paralelamente, favorecer ou prejudicar a relação entre professores e alunos, o presente estudo elegeu focalizar as habilidades sociais educativas de docentes e a síndrome de Burnout.

\section{As Habilidades Sociais Educativas do Professor}

Alguns estudos caracterizam o perfil de um professor "resiliente" como aquele profissional que dedica certo tempo para adquirir habilidades necessárias para atuar com eficácia (Achkar, 2013; Fajardo, 2015; Machado et al., 2014; Yunes et al., 2015). Este educador, que investe em capacitação e formação continuada colabora na construção de processos de resiliência no ambiente escolar, pois transmite suas expectativas, age com afeto, apoio e oferece oportunidades de participação (Achkar, 2013; Fajardo, 2015; Henderson \& Milstein; 2003; Machado et al., 2014; Yunes et al., 2015). Além disso, se esforça para ensinar habilidades para a vida, determinando limites claros que enriquecem e fortalecem os vínculos sociais (Achkar, 2013; Henderson \& Milstein, 2003).

Nesse sentido, Coimbra e Fontaine (2015) indicam funções primordiais exercidas pelas habilidades sociais em relação a processos de resiliência e que funcionam como fatores de proteção mediante as situações adversas. Isto porque, indivíduos mais habilidosos, por serem mais ajustáveis e flexíveis, encontram-se em posição de vantagem diante de situações de risco (Coimbra \& Fontaine, 2015; Fergus \& Zimmerman, 2005). Além disso, esses mesmos indivíduos são capazes de responder mais positivamente àqueles que estão a sua volta e acabam por exercer importantes papéis de proteção e apoio, junto às famílias, colegas e comunidade em geral (Coimbra \& Fontaine, 2015; Noltemeyer \& Bush, 2013; Zolkoski \& Bullock, 2012).

Conforme Del Prette e Del Prette (2001), o conceito de Habilidades Sociais Educativas (HSE) pertencente ao campo teórico-prático das Habilidades Sociais e refere-se àquelas habilidades que intencionam a promoção do desenvolvimento e da aprendizagem do outro, de maneira formal ou informal. Del Prette e Del Prette (2001, 2008) 
partem da premissa que entre professor e aluno há comportamentos considerados determinantes da educação formal e destacam quatro classes específicas da ação docente: (a) habilidade em apresentar o conteúdo, explicar e motivar a aprendizagem; (b) habilidade em transmitir informações e conhecimentos com clareza, encorajando a mudança de comportamento positivamente; (c) habilidade em mediar interações educativas entre os alunos, solicitando e valorizando a ajuda mútua; (d) habilidade para avaliar as atividades, e levar os alunos a serem capazes de julgar o próprio desempenho bem como 0 desempenho dos colegas.

Pesquisas afirmam que interações entre professores e alunos no decurso das aulas são importantes para a construção social do conhecimento e há consenso entre os autores que o professor conduz, lidera e media tais interações (Del Prette \& Del Prette, 2008; Gomes, Cecato, Montiel, Martinelli, \& Santos, 2014; Guerra et al., 2015; Soares et al., 2009; Tucci, 2011). Machado et al. (2014) defendem as habilidades interpessoais como competências essenciais para o exercício da docência, pois cabe ao professor fazer uma leitura do ambiente e compreender as singularidades do contexto do qual participa com seus alunos. Essa avaliação contextual permite que 0 professor perceba as demandas prementes a sua volta e atue de maneira flexível de modo a conduzir com sensibilidade e habilidade os conflitos que possam surgir, focando a qualidade do processo de ensino-aprendizagem e a promoção de desenvolvimento humano dos alunos (Guerra et al., 2015). Tucci (2011) verificou em seu estudo que o professor que faz uso das HSE tem a seu favor o uso de estratégias didático pedagógicas que facilitam a interação entre seus alunos e com ele mesmo, colocando em prática atividades que possibilitam a identificação de expressões de emoção, afeto e comportamentos sociais adequados.

Tendo em vista que o professor é um dos responsáveis pela coordenação das relações de alunos, mesmo que indiretamente, diversos estudos vêm sendo realizados com o objetivo de investigar tais elementos na área educacional (Cooper, 2014; DoménechBetoret et al., 2015; Bolsoni-Silva et al., 2013; Gomes et al., 2014; Machado, Yunes, \& Silva, 2014; Silva, Aznar-farias, \& Silvares, 2009). Os resultados desses estudos evidenciam atitudes docentes socialmente hábeis como importantes indicativos de diminuição de dificuldades de comportamento e de interferências na aprendizagem, sinalizando a relevância das HSE para a qualidade das relações entre os docentes e seus educandos. 


\section{A Síndrome de Burnout}

O contato direto com o público e, muitas vezes, o exercício diário em condições inadequadas, faz com que os professores sejam um grupo vulnerável a apresentar estados iniciais de estresse até a manifestação da denominada síndrome de Burnout (Achkar, 2013; Benevides-Pereira, 2008; Dalagasperina \& Monteiro, 2014; Mesquita et al., 2013; Silva, Bolsoni-Silva, Rodrigues, \& Capellini, 2015). A partir de uma concepção psicossocial e multidimensional, a síndrome de Burnout decorre da tensão emocional crônica que surge pelo contato excessivo com outras pessoas e compreende três dimensões (Maslach et al., 2001). A primeira é a exaustão emocional que é caracterizada por uma sensação de sentir-se emocionalmente sobrecarregado e mentalmente esgotado. A segunda é a despersonalização que se refere a uma atitude negativa e de indiferença para com as pessoas com quem se trabalha que são sujeitos da sua ação e recebem o seu serviço, levando a relações interpessoais desprovidas de afeto. A terceira dimensão é uma reduzida realização pessoal que indica um sentimento de desencanto com o trabalho, com uma consequente autoavaliação negativa de suas habilidades e competências para a execução dessas tarefas, 0 que acarreta comprometimento profissional (Maslach et al., 2001).

Estudos revelaram que elevados índices da síndrome de Burnout entre os docentes são associadas ao excesso de horas em sala de aula, o número de alunos por turma, condições laborais inadequadas, sobrecarga de provas e exercícios para corrigir (Andrade \& Cardoso, 2012; Dalagasperina \& Monteiro, 2014; Doménech-Betoret et al., 2015; Gomes et al., 2014). Dalagasperina e Monteiro (2014) ao investigar fatores do estresse laboral sobre a síndrome de Burnout, com 202 professores de vários níveis de ensino da rede particular, encontraram associações positivas entre a falta de organização do trabalho e dificuldades na relação com os alunos. Na mesma direção, Weber, Leite, Stasiak, Santos e Forteski (2015) encontraram, com 270 professores da rede pública de ensino de diferentes níveis, que a ausência de apoio pelos diretores e problemas de disciplina e motivação dos alunos eram as fontes mais relevantes de estresse no contexto de trablho. Desse modo, o Burnout pode enfraquecer sentimentos de competência pessoal e prejudicar as relações entre 0 professor e seus alunos (Bolsoni-Silva et al., 2013). No entanto, a literatura acerca das percepções dos professores sobre seus recursos pessoais promotores de resiliência ainda é incipiente (Fajardo, Minayo, \& Moreira, 2013; Malloy \& Allen, 2007; Raymundo \& Leão, 2014; Yunes et al., 2015).

A partir da revisão de literatura foi possível verificar que as habilidades sociais educativas nos professores podem possibilitar melhor interação com seus alunos, oportunizando aos discentes um 
desempenho escolar e socioafetivo mais positivo (Bolsoni-Silva et al., 2013; Tucci, 2011). Dados do relatório sobre o Índice de Desenvolvimento da Educação Básica (Ideb, 2013) evidenciaram que um total aproximado de 473.435 estudantes que frequentavam os Anos Finais do E.F. abandonou a escola durante o ano de 2013. Desse modo, parece pertinente focalizar esse período escolar para buscar identificar fatores relacionados à permanência e à conclusão do E.F. e também ao ingresso no Ensino Médio. Contudo, não foram identificados estudos que procurassem investigar as possíveis influências das habilidades sociais educativas dos professores e da síndrome de Burnout sobre a qualidade da relação do professor e seus alunos nos anos finais do E.F. Para Guerra et al. (2015), discutir as práticas educativas de professores e a presença de habilidades sociais educativas é relevante, visto que as interações que ocorrem na escola podem determinar ou manter comportamentos indesejáveis e déficits nas habilidades sociais dos alunos. Dessa forma, se os contextos dos alunos forem respeitados para um desempenho escolar mais produtivo, é possível favorecer a comunicação interpessoal e criar um ambiente relacional de desenvolvimento entre professores e alunos (Del Prette \& Del Prette, 2008; Yunes et al., 2015). Assim, este estudo teve como objetivo correlacionar as habilidades sociais educativas dos professores, o Burnout e a relação professor-aluno nos anos finais do Ensino Fundamental.

\section{Método}

\subsection{Participantes}

Foi realizado um estudo preditivo com uma amostra selecionada por conveniência. Participaram da pesquisa 400 professores, com idade média de 36,08 anos ( $D P=9,94$ ), de ambos os sexos (348 mulheres e 52 homens) que lecionavam para o $70(n=165), 8^{\circ}(n=136)$ e $9^{\circ}$ $(\mathrm{n}=99)$ ano do Ensino Fundamental, em oito escolas, quatro públicas e quatro particulares de três cidades do Estado do Rio de Janeiro. Conforme recomendação de Tabashnick e Fidell (2001), para controlar as influências do tipo de escola (pública e particular) na relação professor-aluno, assim como para aumentar a capacidade de generalização dos resultados, a amostra foi composta de forma equilibrada por professores de escolas públicas $(n=200)$ e particulares $(n=200)$. Como critério de inclusão, os professores deveriam ensinar nos três últimos anos do E.F. das escolas que aceitaram participar do estudo. Como mencionado anteriormente, os Anos Finais do E.F. caracterizam-se como um momento crítico na trajetória escolar devido aos elevados níveis de abandono pelos alunos (Ideb, 2013). 
Em relação ao tempo de docência 198 (49,5\%) professores informaram que lecionam entre 0 a 10 anos, 129 (32,3\%) de 11 a 20 anos e os demais $73(18,3 \%)$ por mais de 21 anos. Em relação a formação, $285(71,35 \%)$ professores tinham graduação e os demais pós-graduação (28,8\%). No que diz respeito ao nível socioeconômico, $0,5 \%$ pertenciam a classe $D, 29,3 \%$ à classe $C(1$ e 2$), 61 \%$ à classe $B(1$ e 2$)$ e $9,3 \%$ à Classe $A$ ( 1 e 2 ). Todas as escolas incluídas no estudo atendiam somente alunos dos anos finais do E.F. (do $6^{\circ}$ ao $9^{\circ}$ ano). Três delas situavam na cidade de São Gonçalo (duas na região central e uma na periferia), três na cidade de Itaboraí (na região central) e as outras duas na cidade de Niterói (na região central).

\subsection{Instrumentos}

Questionário de informações demográficas. Elaborado para este estudo no intuito de coletar informações sociais e demográficas: (a) informações gerais de aplicação; (b) informações sobre os participantes (nome, idade, sexo, ano que leciona, tempo que leciona, disciplina e formação). Foi também empregado o Questionário de Classificação Econômica Brasil (ABEP, 2011) que identifica o nível socioeconômico e permite a estratificação das famílias, em ordem decrescente de poder aquisitivo, em cinco classes: A (A1 e A2), B (B1 e B2), C, D e E.

Escala da relação Professor-aluno. É um instrumento desenvolvido por Del Prette e Del Prette (prelo) que avalia a qualidade da relação do professor com seus alunos em sala de aula. É composto por 7 itens dispostos em uma escala tipo Likert que varia que -5 a +5 , sendo que no presente estudo os itens foram codificados numa escala de 1 a 10: (1) Qualidade geral da relação; (2) Diálogo com os alunos; (3) Respeito dos alunos por mim; (4) Afetividade dos alunos por mim; (5) Qualidade da minha prática; (6) Aprendizagem dos alunos; (7) Disciplina dos alunos em classe. O índice de consistência interna encontrado na escala para a presente amostra foi $a=0,93$.

Inventário de Habilidades Sociais Educativas - versão professores (IHSE-Pr, Del Prette, prelo). É um inventário de autorrelato com 64 itens que descrevem comportamentos sociais apresentados na relação com os alunos, respondidos pelos professores em uma escala Likert que varia de Nunca ou Quase Nunca (0) a Sempre ou Quase Sempre (4). Os itens da escala foram elaborados a partir do Sistema de Categorias de Habilidades Sociais Educativas, proposto por Del Prette \& Del Prette (2008) que comporta duas escalas: (1) Organizar Atividade Integrativa (14 itens), por exemplo, "Altero o ambiente da sala de aula com tapetes, almofadas, traços de giz no chão etc. e/ou levo os alunos para atividades em outros ambientes (biblioteca, pátio, horta, rua etc"); (2) Habilidades de Conduzir atividade interativa (50 itens), por exemplo, "Apresento modelo de comportamentos sociais 
(forma de falar, gentileza, paciência etc.) que espero sejam reproduzidos pelos alunos". Foram encontrados índices de consistência internas para as escalas (variou de $a=0,84$; à $a=$ $0,87)$ e para o escore total do instrumento $(a=0,94)$.

Inventário de Burnout de Maslach (MBI, Carlotto \& Câmara, 2004). É utilizado para a avaliação da síndrome de Burnout no trabalhador. Esse instrumento é disponibilizado pela editora Mind Garden (www.mindgarden.com) que tem os direitos autorais dos inventários para avaliação do Burnout de Maslach. Os autores adquiriram a licença ( $n^{\circ}$ JODVZDGAQ) para aplicação do inventário e publicação dos resultados. Foi validado para uma amostra de professores brasileiros do Ensino Fundamental, Médio e Superior por Carlotto e Câmara (2004). O instrumento contém 22 itens com respostas distribuídas numa escala tipo Likert de cinco pontos (nunca $=1 \mathrm{a}$ diariamente $=1$ ), com três dimensões com os seguintes índices de consistência interna para a presente amostra: Exaustão ( $a=0,86$, "Sinto que meu trabalho está me desgastando"); Realização profissional ( $a=0,87$, "Sinto que estou exercendo influência positiva na vida das pessoas, através de meu trabalho"); Despersonalização ( $a=0,68$, "Sinto que me tornei mais duro com as pessoas, desde que comecei este trabalho").

\subsection{Procedimentos}

\subsubsection{Coleta de dados}

A pesquisa foi aprovada, de acordo com o Parecer $N^{\circ} 921.458 / 2014$ CAAE: 39701814.5.0000.5289, pelo Comitê de Ética em Pesquisa da Universidade onde se realizou o estudo. Primeiramente, a pesquisadora entrou em contato com as direções das instituições de ensino para a apresentação do estudo e obtenção da autorização para a aplicação dos instrumentos. A coleta de dados ocorreu na sala dos professores em formato de aplicação em grupo, com preenchimento individual e compartilhando o mesmo espaço, no horário combinado com os mesmos.

Os objetivos e procedimentos, bem como a livre participação, foram informados aos docentes mediante a entrega e assinatura do Termo de Consentimento Livre e Esclarecido. Também foi apresentada a garantia de confidencialidade dos dados coletados e reiterada a participação voluntária na mesma que, mediante consulta verbal, aceitaram participar da aplicação dos instrumentos (duração média 50 minutos). 


\subsubsection{Análise de Dados}

A análise de dados foi executada com o software Statistical Package for the Social Sciences for Windows (SPSS, versão 22.0). Primeiramente, calculou-se a correlação entre as variáveis do estudo (coeficiente $r$ de Pearson). Para interpretar os valores dos coeficientes de correlação ( $r$ ), utilizou-se a classificação proposta por Bryman e Cramer (2003): $<0,20=$ muito fraca; $\geq 0,20$ e $<0,40=$ fraca; $\geq 0,40$ e $<0,70=$ moderada; $\geq 0,70$ e $<0,90=$ forte; $>0,90$ $=$ muito forte.

$\mathrm{Na}$ sequência, foram testados e confirmados os pressupostos de normalidade, independência e multicolinearidade e, em seguida, procedeu-se a análise de regressão linear múltipla (método stepwise). A relação professor-aluno foi a variável dependente. Foram consideradas como variáveis independentes o total das Habilidades Sociais Educativas dos Professores e o total do Burnout.

\section{Resultados}

Os resultados indicam correlações positivas (fraca) entre as Habilidades Sociais Educativas (HSE) dos professores e a relação professor-aluno $(r=0,36 ; p=0,001)$. São encontradas correlações negativas (fracas) entre a síndrome de Burnout e a relação professoraluno $(r=-0,28 ; p=0,001)$. Também são indicadas associações negativas (fraca) entre as HSE dos professores e síndrome Burnout ( $r$ $=-0,23 ; p=0,001)$.

A Tabela 1 apresenta as variáveis preditoras para a relação professor-aluno. O modelo significativo $(F(2,39)=42,65, p=0,001)$ apresentado na Tabela 1 mostra que as HSE dos professores e a síndrome de Burnout explicaram $17 \%$ da variabilidade da relação professor-aluno ao final do Ensino Fundamental. As HSE dos professores foi a variável preditora com maior peso sobre as relação professor-aluno, isto é, quanto mais HSE melhor é a relação entre professor e aluno. 
Tabela 1

Modelo de regressão para a predição da relação professor-aluno nos anos finais do Ensino Fundamental

\begin{tabular}{|c|c|c|c|c|c|c|}
\hline Preditores & $\begin{array}{l}\text { Cons- } \\
\text { tante }\end{array}$ & $B$ & EP B & $\beta$ & $95 \%$ IC & $R^{2}$ \\
\hline \multicolumn{7}{|l|}{ Habilidades } \\
\hline $\begin{array}{l}\text { Sociais } \\
\text { Educativas } \\
\text { dos } \\
\text { Professores }\end{array}$ & $\begin{array}{c}19,73 \\
*\end{array}$ & 0,06 & 0,01 & 0,31 & $\begin{array}{c}{[0,04 ;} \\
0,09]\end{array}$ & $\begin{array}{c}0,13 \\
*\end{array}$ \\
\hline $\begin{array}{l}\text { Síndrome de } \\
\text { Burnout }\end{array}$ & $\begin{array}{c}12,63 \\
*\end{array}$ & $\begin{array}{c}-0,1 \\
0\end{array}$ & 0,02 & $\begin{array}{c}-0,2 \\
1 \\
\end{array}$ & $\begin{array}{l}{[-0,15} \\
-0,06]\end{array}$ & $\begin{array}{c}0,17 \\
*\end{array}$ \\
\hline \multicolumn{7}{|c|}{$\begin{array}{l}\text { Nota. } N=400 . B=\text { coeficiente de regressão não padronizado, EP } B= \\
\text { Erro Padrão do coeficiente de regressão não padronizado; } \beta= \\
\text { coeficiente de regressão padronizado; } I C=\text { Intervalo de Confiança; } R^{2}= \\
\text { coeficiente de determinação ajustado. } \\
* p<0,001 \text {. }\end{array}$} \\
\hline
\end{tabular}

\section{Discussão}

Os estudos sobre processos de resiliência no contexto educativo têm focalizado os alunos em detrimento da figura do professor. Assim, as análises acerca do desenvolvimento e de fatores de proteção que incidem sobre os riscos, são mais frequentemente pesquisadas em populações de estudantes (Fajardo et al., 2013; Malloy \& Allen, 2007; Raymundo \& Leão, 2014; Yunes et al., 2015). Além disso, não foram identificados relatos de pesquisas que buscassem investigar possíveis associações entre as habilidades sociais educativas (HSE) dos professores, as expressões relacionadas à síndrome do Burnout e a relação professor-aluno nos Anos Finais do Ensino Fundamental.

Os resultados do presente estudo indicaram que quanto mais HSE dos professores melhor são os indicadores de relação saudável entre mestres e alunos. Inversamente, quanto maiores os níveis de Burnout pior é a qualidade da relação professor-aluno e menor a frequência de HSE dos professores. Estes resultados são corroborados por outras pesquisas (Guerra et al., 2015; Tucci, 2011) os quais identificaram professores que utilizam de um elaborado repertório de HSE são mais capazes de facilitar as relações interpessoais no contexto escolar.

De maneira inversa, conforme as correlações negativas encontradas e em consonância com estudo anterior de Silva et al. (2015), déficits em HSE influenciam negativamente nos níveis de Burnout em professores, o que prejudica a relação com os alunos. Nessa direção, estudos recentes (Doménech-Betoret et al., 2015) têm indicado que o papel de docente como mediador promove relacionamentos satisfatórios entre professor-aluno, sendo um recurso que exerce 
suporte para maior engajamento do professor na sua tarefa de educar integralmente.

A figura de professor-mediador pode funcionar como elemento de proteção que resulta em interações positivas e de confiança entre professor-aluno, gerando mudanças no repertório cognitivo e comportamental dos educandos. Este papel mediador pode, inclusive, ter o efeito de neutralizar/minimizar o Burnout, pois fortalece as capacidades e características individuais dos docentes. Além disso, os professores tornam o contexto educativo mais flexível para lidar com as mudanças não lineares e inesperadas que surgem como parte do processo de ensino-aprendizagem (Buschbacher, 2014).

O modelo testado pela regressão linear múltipla constatou que as HSE dos professores foi a variável com maior peso na variabilidade da relação professor-aluno. Esse resultado encontra apoio na literatura (Del Prette \& Del Prette, 2008; Guerra et al., 2015; Gomes et al., 2014; Soares et al., 2009; Tucci, 2011), visto que as HSE dos professores, no transcorrer das aulas são importantes, por exemplo, durante a exposição de um novo conteúdo acadêmico.

A presença de HSE dos professores pode promover relações professor-aluno caracterizadas pelo diálogo, respeito e afetividade e não apenas pela preocupação com a transmissão de conteúdo acadêmicos (Cooper, 2014; Dotterer \& Lower, 2011; Machado et al., 2014). Portanto, as HSE dos professores podem favorecer a construção de um ambiente escolar harmonioso que desperte nos alunos e nos professores, o desejo de fazer parte das atividades de ensino-aprendizagem (Paiva \& Del Prette, 2009), assim como facilita o surgimento de um senso de pertencimento à comunidade escolar.

Nesse sentido, os dados revelaram que as HSE dos professores se tornam primordiais para o exercício competente da profissão (Machado et al., 2014; Gomes et al., 2014; Doménech-Betoret et al., 2015). Isto porque, além da preocupação com a qualidade do conteúdo ensinado, o professor precisa estar focado na promoção ética-moral de seus alunos (Guerra et al., 2015) e, ao mesmo tempo, deve demonstrar ter metas e objetivos definidos sobre 0 desenvolvimento dos alunos como seres humanos, tornando possível a realização das tarefas propostas (Fontes \& Azzi, 2012). Machado et al. (2014) afirmam que professores que agem com sensibilidade e se posicionam como um porto seguro para os alunos, favorecem a confiança dos alunos para explorar o ambiente da escola e para participar das atividades de aprendizagem. Ao agirem dessa forma, os professores passam a atuar como "tutores de resiliência" (Cyrulnik, 2005; Machado et al., 2014).

$\mathrm{Na}$ atualidade, excessivas questões tecnoburocráticas estão sendo associadas a obrigações que devem ser cumpridas pelos professores (Dalagasperina \& Monteiro, 2014). Estes, por sua vez, tem dificuldades de encontrar meios para realizar suas tarefas por 
vocação e carisma, características que retornavam para o professor em forma de satisfação pessoal e profissional (Achkar, 2013). Sendo assim, a presença do Burnout constitui-se como um fator de risco, podendo desencadear danos e equívocos no preparo e na elaboração das aulas, na motivação para ministrá-las, na capacidade criativa e flexível de lidar com situações adiversas e na habilidade de exercer a empatia, distanciando o professor de seus alunos (Silva et al., 2015). No entanto, as variáveis de apoio social internas da escola (professores e colegas) ou externas (amigos, parceiros e famíliares) e de satisfação no trabalho relacionam-se de forma inversa com as dimensões do Burnout (Dalagasperina \& Monteiro, 2014; Weber et al., 2015). Dessa forma, recursos internos, a exemplo das HSE dos professores e recursos externos como o apoio da direção escolar funcionam podem amenizar o impacto dos efeitos que levam a síndrome de Burnout, indicando que esses recursos auxiliam na redução dos níveis de adoecimento do professor (Doménech-Betoret et al., 2015).

\section{Considerações finais}

O presente estudo contribuiu para evidenciar como as habilidades sociais educativas de professores (HSE) e a síndrome de Burnout impactam sobre a relação professor-aluno nos Anos Finais do E.F. Avaliar as influências da associação entre as HSE dos professores e do Burnout sobre a qualidade da relação professor-aluno, pode indicar caminhos para a promoção de programas de intervenção que facilitem o surgimento de processos de resiliência no contexto educativo. A presente pesquisa também contribuiu para a compreensão das influências de variáveis pessoais e do contexto escolar que atuam como risco e proteção na profissão docente nos anos finais do E.F., sinalizando para a importância de um ambiente relacional de qualidade entre professores e alunos.

$\mathrm{O}$ estudo apresentou algumas limitações. Primeiro, refere-se ao fato de ter utilizado uma amostra selecionada por conveniência, pertencente a algumas escolas públicas e privadas situadas no estado do Rio de Janeiro. Segundo, os dados foram coletados a partir das percepções dos professores e, desse modo, seria interessante que futuras pesquisas incluíssem informações coletadas a partir de com outros informantes, como, a equipe de apoio pedagógica e diretiva. Terceiro, o coeficiente de determinação do modelo final foi pequeno $(17 \%)$, sugerindo que outras variáveis podem estar associadas à qualidade da relação professor-aluno. Apesar destas limitações, este estudo amplia os resultados de investigações anteriores no campo das pesquisas em resiliência em espaços escolares. 
Ressalta-se que, quando se trata de atuação docente, questões como salários adequados, segurança pública e escolas em boas condições de trabalho merecem ser tratadas por instâncias públicas nos âmbitos educacional, municipal, estadual e federal, o que vai muito além de docentes apresentarem elaborados repertórios de HSE. Por fim, destaca-se que este trabalho não esgota as possibilidades de investigação sobre o tema e sugere que novas pesquisas sejam realizadas de modo a incluir outras variáveis tais como o clima escolar e a relação escola, família e comunidade ao comportamento dos alunos.

\section{Referências}

Achkar, A. E. (2013). Resiliência, ferramenta para uma educação de qualidade. Curitiba: Appris.

Andrade, P. S., \& Cardoso, T. A. de O. (2012). Prazer e dor na docência: Revisão bibliográfica sobre a síndrome de burnout. Saúde e Sociedade, 21(1), 129-140. http://dx.doi.org/10.1590/S0104-12902012000100013

Benevides-Pereira, A. M. T. (2008). Estrés y la psicologia positiva in las emociones positivas. Madrid: Pirámide.

Bolsoni-Silva, A. T., Verdu, A. C. M. A., Carrara, K., Melchiori, L. E., Leite, L. P., \& Calais, S. L. (2013). Ampliando comportamentos pró-éticos dos alunos: Relato de pesquisa e intervenção com educadores do Ensino Fundamental. Temas em Psicologia, 21(2), 347-359. http://dx.doi.org/10.9788/TP2013.2-04

Brasil. Ministério da Educação (2013). Índice da Educação Básica. Brasília: Ideb. Retrieved from http://ideb.inep.gov.br/resultado

Bryman, A., \& Cramer, D. (2003). Análise de dados em ciências sociais, introdução às técnicas utilizando o SPSS para Windows. Oeiras: Celta Editora.

Buschbacher, R. (2014). A teoria da resiliência e os sistemas socioecológicos: Como se preparar para um futuro imprevisível? Boletim regional, urbano e ambiental, 9, 12-24. Retrieved from http://repositorio.ipea.gov.br/bitstream/11058/5561/1/BRU_n0 9_teoria.pdf

Carlotto, M. S., \& Câmara, S. G. (2004). Análise fatorial do Maslach Burnout Inventory (MBI) em uma amostra de professores de instituições particulares. Psicologia em Estudo (Maringá), 9(3), 499-505.

73722004000300018

http://dx.doi.org/10.1590/S1413-

Carlotto, M. R., Dias, S. R. S., Batista, J. B. V., \& Diehl, L. (2015). O papel mediador da autoeficácia na relação entre a sobrecarga de trabalho e as dimensões de Burnout em professores. Psico- 
USF, 20(1), 741-752. http://dx.doi.org/10.1590/141382712015200102

Coimbra, S. M. G., \& Fontaine, A. M. V. G. (2015). Resiliência e habilidades sociais: Reflexões conceituais e práticas para uma nova geração. In Z. A. P. Del Prette, A. B. Soares, C. S. PereiraGuizzo, M. F. Wagner \& V. B. R. Leme (Orgs.), Habilidades Sociais: Diálogos e intercâmbios sobre pesquisa e prática (pp. 186-220). Novo Hamburgo: Sinopsys.

Cooper, S. K. (2014). Eliciting engagement in the high school classroom: A mixed-methods examination of teaching practices. American Educational Research Journal, 51(2), 363-402. http://dx.doi.org/10.3102/0002831213507973

Cyrulnik, B. (2005). O murmúrio dos fantasmas. São Paulo: Martins Fontes.

Dalagasperina, P., \& Monteiro, J. K. (2014). Preditores da síndrome de burnout em docentes do ensino privado. Psico-USF, 19(2), 265-275. http://dx.doi.org/10.1590/1413-82712014019002011

Del Prette, Z. A. P., \& Del Prette, A. (2001). Psicologia das relações interpessoais: Vivências para o trabalho em grupo. Petrópolis: Vozes.

Del Prette, A., \& Del Prette, Z. A. P. (2008). Um sistema de categorias de habilidades sociais educativas. Paidéia (Ribeirão Preto), 18(41), 517-30. http://dx.doi.org/10.1590/S0103$863 \times 2008000300008$

Del Prette, Z. A. P., \& Del Prette, A. (prelo). Inventário de habilidades Sociais Educativas - versão Professor (IHSE-Prof).

Doménech-Betoret, F., Lloret-Segura, S., \& Gómez-Artiga, A. (2015). Teacher support resources need satisfaction and well-being. Spanish Journal of Psychology, 18(6), 1-12. http://dx.doi.org/10.1017/sjp.2015.8

Dotterer, A. M., \& Lowe, K. (2011). Classroom context, school engagement, and academic. Achievement in early adolescence. Journal Youth Adolescence, 40(1), 1649-1660. http://dx.doi.org/10.1007/s10964-011-9647-5

Facci, M. G. D., Tessaro, N. S.; Leal, Z. F. R. G., Silva, V. G., \& Roma, C. G. (2007). Psicologia histórico-cultural e avaliação psicológica: $O$ processo ensino-aprendizagem em questão. Revista Semestral da Associação Brasileira de Psicologia Escolar e Educacional (ABRAPEE), 11(2), 323-338. http://dx.doi.org/10.1590/S1413-85572007000200011

Fajardo, I. N. (2015). Resiliência e Educação: Exemplo das Escolas do Amanhã. Curitiba: Appris.

Fajardo, I. N., Minayo, M. C. S., \& Moreira, C. O. F. (2013). Resiliência e prática escolar: Uma revisão crítica. Revista: Educação \& Sociedade, 34(122), 213-224. http://dx.doi.org/10.1590/S0101-73302013000100012 
Fergus, S., \& Zimmerman, M. A. (2005). Adolescent resilience: A framework for understanding healthy development in the face of risk. Annual Review of Public Health, 26(1), 399-419. http://dx.doi.org/10.1146/annurev.publhealth.26.021304.1443 57

Fernandes, L. M., \& Leme, V. B. R. (2014). Transição do Ensino Fundamental para o Ensino Médio: A importância das habilidades sociais e do apoio social dos estudantes. In L. Campos (Org.), Resiliência e Habilidades sociais: Reflexões acerca das suas articulações e seus desdobramentos na escola e na vida (pp. 41-62). Curitiba: Appris.

Fletcher, D., \& Sarkar, M. (2013). Psychological resilience: A review and critique of definitions, concepts and theory. European Psychologist, 18(1), 12-23. http://dx.doi.org/10.1027/10169040/a000124

Fontes, A. P., \& Azzi, R. G. (2012). Crenças de autoeficácia e resiliência: Apontamentos da literatura sociocognitiva. Estudos de Psicologia (Campinas), 29(1), 105-114. http://dx.doi.org/10.1590/S0103-166X2012000100012

Garcia, S. C., Brino, R., \& Williams, L. C. A. (2009). Risco e resiliência em escolares: Um estudo comparativo com múltiplos instrumentos. Psicologia da Educação, 28(1), 23-49. Retrieved from

http://pepsic.bvsalud.org/scielo.php?script=sci_arttext\&pid=S1 414-69752009000100003\&lng=pt\&nrm=iso\&tlng=pt

Gomes, A. J. S., Cecato, J. F., Montiel, J. M., Martinelli, J. E., \& Santos, G. C. (2014). A influência das habilidades sociais nos processos educacionais. Perspectivas Médicas, 25(1), 20-26. Retrieved from http://www.redalyc.org/articulo.oa?id=243230610004

Guerra, B. T., Rovaris, J. A., Mariano, M., Guidugli, P. M., Rosanti, S., \& Bolsoni-Silva, A. T. (2015). Análise das queixas da rede municipal encaminhadas para a Educação Especial. Psicologia Escolar e Educacional, 19(2), 321-328. http://dx.doi.org/10.1590/2175-3539/2015/0192841

Henderson, N., \& Milstein, M. M. (2003). Resiliency in schools: Making it happen for students and educators. California: Corwin Press.

Juliano, M. C. C., \& Yunes, M. A. M. (2014). Reflexões sobre rede de apoio social como mecanismo de proteção e promoção de resiliência. Ambiente \& Sociedade, 27(3), 135-152. http://dx.doi.org/10.1590/S1414-753X2014000300009

Machado, J. A., Yunes, M. A., \& Silva, G. F. (2014). A formação continuada de professores em serviço na perspectiva da abordagem ecológica do desenvolvimento humano. 
Contrapontos,

14(3),

512-526.

http://dx.doi.org/10.14210/contrapontos.v14n3.p512-526

Malloy, W. W., \& Allen, T. (2007). Teacher retention in a teacher resiliency-building rural school. The Rural Educator, 28(2), 1927. Retrieved

http://files.eric.ed.gov/fulltext/EJ783870.pdf

from

Maslach, C., Schaufeli, W. B., \& Leiter, M. P. (2001). Job Burnout. Annual Review Psychology, 52(1), 397-422. http://dx.doi.org/10.1146/annurev.psych.52.1.397

Masten, A. S. (2014). Global perspectives on resilience in children and youth. Child Development, 85(1), 6-20. http://dx.doi.org/10.1111/cdev.12205

Mattos, A. R., Pérez, B. C., Almada, C. V. R., \& Castro, L. R. (2013). $O$ cuidado na relação professor-aluno e sua potencialidade política. Estudos de Psicologia (Natal), 18(2), 369-377. http://dx.doi.org/10.1590/S1413-294X2013000200024

Meireles, R. M. (2009). As relações entre as medidas de habilidades sociais do professor do Ensino Fundamental II e seu desempenho social em sala de aula. Revista Visões, 1(6), 1-28. Retrieved from http://www.fsma.edu.br/visoes/ed06/Edicao_6_artigo_3.pdf

Mesquita, A. A., Gomes, D. S., Lobato, J. L., Gondim, L., \& Souza, S. B. (2013). Estresse e síndrome de burnout em professores: Prevalência e causas. Psicologia Argumento (Curitiba), 31(75), 627-635. Retrieved from http://www2.pucpr.br/reol/pb/index.php/pa?dd1=12630\&dd99 $=$ view $\&$ dd $98=\mathrm{pb}$

Noltemeyer, A. L. (2013). SPI's virtual special issue 'Resilience Across Contexts'. School Psychology International, 34(5), 473-485. http://dx.doi.org/10.1177/0143034313505520

Noltemeyer, A. L., \& Bush, K. R. (2013). Adversity and resilience: A synthesis of international research. School Psychology International, $34(2)$,

474-487. http://dx.doi.org/10.1177/0143034312472758

Paiva, M. L. M. F., \& Del Prette, Z. A. P. (2009). Crenças docentes e implicações para o processo de ensino-aprendizagem. Psicologia Escolar e Educacional, 13(1), 75-85. Retrieved from http://www.scielo.br/pdf/pee/v13n1/v13n1a09.pdf

Raymundo, R. S., \& Leão, M. A. B. G. (2014). Resiliência e educação: Um Panorama dos Estudos Brasileiros. Revista Ciências Humanas - UNITAU (Taubaté), 7(2), 76-98. http://www.rchunitau.com.br/index.php/rch/article/view/158/8 5

Silva, D. A., Aznar-Farias, M., \& Silvares, E. F. M. (2009). Influência do treino em habilidades sociais na relação professor-aluno: Uma contribuição psicopedagógica para professores. Revista 
ACOALFAplp: Acolhendo a Alfabetização nos Países de Língua portuguesa, São Paulo, 3(5), 68-90. http://dx.doi.org/10.11606/issn.1980-7686.v3i5p68-90

Silva, N. R., Bolsoni-Silva, A. T., Rodrigues, O. M. P. R., \& Capellini, V. L. M. F. (2015). O trabalho do professor, indicadores de burnout, práticas educativas e comportamento dos alunos: Correlação e predição. Revista Brasileira de Educação Especial (Marília), 21(3), 363-376. http://dx.doi.org/10.1590/S141365382115000300004

Soares, A. B., Naiff, L. M. A., Fonseca, R. B., Cardozo, A., \& Baldez, M. O. (2009). Estudo comparativo de habilidades sociais e variáveis sociodemográficas de professores. Psicologia: Teoria e Prática, 11(1), 35-49. Retrieved from http://pepsic.bvsalud.org/scielo.php?script=sci_arttext\&pid=S1 516-36872009000100004

Tabashnick, B. G., \& Fidell, L. S. (2001). Using multivariate statistics. 4th Ed. Needham Heights, MA: Allyn \& Bacon.

Tucci, C. H. C. (2011). A importância das Habilidades Sociais Educativas na perspectiva de professores de alunos sem ou com necessidades educacionais especiais (Dissertação de Mestrado). Universidade Federal de São Carlos. São Carlos, São Paulo, Brasil.

Ungar, M. (2012). The social ecology of resilience: A handbook of theory and practice. New York: Springer.

Weber, L. N. D., Leite, C. R., Stasiak, G. R., Santos, C. A. S., \& Forteski, R. (2015). O estresse no trabalho do professor. Imagens da Educação, 5(3), 40-52. http://dx.doi.org/10.4025/imagenseduc.v5i3.25789

Yunes, M. A. M., Pietro, A. T., Silveira, S. B., Juliano, M. C., \& Garcia, N. M. (2015). Um educador para proteger do risco e tricotar a resiliência: O profissional da educação como agente de proteção e de promoção de resiliência. In S. Cabral \& B. Cyrulnik (Orgs.), Resiliência: Como tirar leite de pedra (p. 155-170). São Paulo: Casa do Psicólogo.

Zolkoski, S. M., \& Bullock, L. M. (2012). Resilience in children and youth: A review. Children and Youth Services Review Journal, 34(1),

2295-2303.

http://dx.doi.org/doi:10.1016/j.childyouth.2012.08.009

\section{Endereço para correspondência \\ Ana Maria Nunes El Achkar}

Faculdade Conecista de Itaboraí - FACNEC

Rua Presidente Costa e Silva, 212, Centro, CEP 24888-055, Itaboraí - RJ, Brasil

Endereço eletrônico: anaelachkar@yahoo.com.br

Vanessa Barbosa Romera Leme

Universidade do Estado do Rio de Janeiro - UERJ 
Rua São Francisco Xavier, 524, $10^{\circ}$ andar, sala 10.006, Bloco D, Maracanã, CEP 20550-900, Rio de Janeiro - RJ, Brasil

Endereço eletrônico: vanessaromera@gmail.com

\section{Adriana Benevides Soares}

Universidade Salgado de Oliveira - UNIVERSO

Rua Marechal Deodoro, 2017, Bloco A, Centro, CEP 24030-060, Niterói - RJ, Brasil

Endereço eletrônico: adribenevides@gmail.com

\section{Maria Angela Mattar Yunes}

Centro Universitário La Salle Canoas - Unilasalle

Avenida Victor Barreto, 2288, CEP 92010-000, Canoas - RS, Brasil

Endereço eletrônico: mamyunes@yahoo.com.br

Recebido em: 18/12/2015

Reformulado em: 25/07/2016

Aceito para publicação em: 25/07/2016

\section{Notas}

* Pedagoga, Doutora e Mestre em Psicologia Social pela Universidade Salgado de Oliveira (UNIVERSO). Coordenadora das Pós-Graduações Lato Sensu em Psicopedagogia e Gestão Escolar (FACNEC). Diretora científica da Coleção Psico \& Pedagógicos da Editora Appris.

** Psicóloga (Unesp-Bauru), Mestre (Psicologia do Desenvolvimento e Aprendizagem - Unesp-Bauru), Doutora (FFCLRP/USP) e Pós-Doutora (UFSCar). Professora Adjunta do Departamento de Fundamentos de Psicologia e do Programa de Pós-Graduação em Psicologia Social da Universidade do Estado do Rio de Janeiro (UERJ).

*** Doutora em Psicologia Cognitiva pela Universidade de Paris XI e Pós-Doutora pela UFSCar. Professora Titular do programa de Pós-Graduação da Universidade Salgado de Oliveira (UNIVERSO), Professora associada da Universidade do Estado do Rio de Janeiro (UERJ).

**** Psicóloga, Mestre em Psicologia do Desenvolvimento pela University of Dundee, Escócia, Doutora em Educação (PUC/SP), Pesquisadora do CNPq. É professora permanente nos Programas de Pós-Graduação em Educação no Centro Universitário La Salle, UNILASALLE/Canoas, RS e Educação Ambiental da Universidade Federal do Rio Grande/FURG. 